\title{
A global model for the occurrence probability of L-band scintillation S4-index
}

\author{
Shih-Ping Chen ${ }^{1}$, Jann-Yenq Liu ${ }^{2,3,4, *}$, Charles C. H. Lin ${ }^{1}$, and Wen-Hao Yeh ${ }^{5}$ \\ ${ }^{I}$ Department of Earth Science, National Cheng Kung University, Tainan City, Taiwan \\ ${ }^{2}$ Center for Astronomical Physics and Engineering, National Central University, Taoyuan City, Taiwan \\ ${ }^{3}$ Department of Space Science and Engineering, National Central University, Taoyuan City, Taiwan \\ ${ }^{4}$ Center for Space and Remote Sensing Research, National Central University, Taoyuan City, Taiwan \\ ${ }^{5}$ National Space Organization, Hsinchu City, Taiwan
}

\section{Article history:}

Received 21 May 2021

Revised 13 July 2021

Accepted 10 August 2021

Keywords:

FORMOSAT-3/COSMIC, FORMOSAT-7/COSMIC-2, Radio occultation, Scintillation S4-index

\section{Citation:}

Chen, S.-P., J.-Y. Liu, C. C. H. Lin, and W.-H. Yeh, 2021: A global model for the occurrence probability of L-band scintillation S4-index. Terr. Atmos. Ocean. Sci., 32, 977-987, doi: 10.3319/TAO.2021.08.10.03

\section{ABSTRACT}

More than 1.4 million S4-index profiles sounded by FORMOSAT-3/COSMIC (F3/C) radio occultation during 2007 - 2014 are used to construct a global scintillation occurrence model for the ground-based GNSS (Global Navigation Satellite System) users. The local maximums of each F3/C S4-index profile at every $10 \mathrm{~km}$ altitude window are integrated to simulate the worst-case L-band S4-index (S4conv) on the ground. The S4conv mega-data bind into $3^{\circ} \times 3^{\circ}$ in latitude $\times$ longitude allows us computing the occurrence probability of the globe for a given S4-index threshold. The occurrence probability of S4 of the developed model agree well with those of ground-based GNSS receivers of the SCINDA (SCIntillation Network Decision Aid) network. Global patterns in the occurrence probability of the model are similar to that of in-situ plasma measurements probed by ROCSAT and FORMOSAT-7/ COSMIC-2 satellites in various solar activities. These agreements and similarities indicate that the constructed empirical model can be employed to calculate and predict L-band S4-index and its occurrence probability in the ionosphere.

\section{INTRODUCTION}

Amplitude scintillations and phase fluctuations caused by plasma density irregularities can disturb radio signals, which have been widely applied on monitoring the quality of telecommunication, positioning, and navigation services. Theoretically, the effect of the radio signal encounters the irregularity follows the phase screen theory (cf. Booker et al. 1950; Hewish 1951) that the signal fading depends on the carrying frequency and the size of the encountered irregularity. Also, the scintillations can be intensified when the signal penetrates multiple phase screens, which is well interpreted by Yeh and Liu (1982) and Carrano and Rino (2016), and therefore, the scintillation occurrence increases with the intensity. Meanwhile, by using long-term observations of ground-based Global Network Satellite System (GNSS) receivers, many empirical regional scintillation models for the L-band $1.5 \mathrm{GHz}$ signals have been developed to forecast

\footnotetext{
* Corresponding author

E-mail:jyliu@jupiter.ss.ncu.edu.tw
}

the scintillation occurrence and intensity as well as to avoid loss-of-lock of signals (cf. Basu et al. 1976; Aarons 1982). Recently, Sreeja and Aquino (2014) analyzed data of GNSS received in Norway during solar peak activity periods of cycle 23 and 24 and developed a L-band scintillation occurrence model for high latitudinal region of European sector. Zhang et al. (2015) used ground-based GNSS receiving data and built an empirical scintillation model over low latitudes of the East Asia sector. Meanwhile, network of SCIntillation Network Decision Aid (SCINDA) constructed by multiple ground-based GNSS receivers is used to monitor scintillation index S4 in low latitudes of the globe. Based on the SCINDA data, Secan et al. (1995) developed the global scintillation model WBMOD (WideBand MODel), which might be one of the most comprehensive scintillation empirical model nowadays. They suggested that the scintillation occurrence is worthwhile to be noticed, and the occurrence simulation should be added in the later version of WBMOD. Therefore, when a S4-index threshold, date, time, and location is specified, the above scintillation models predict the 
intensity and occurrence information. However, groundbased GNSS receivers can simply cover continents, which lack of measurements over the remote and oceanic areas.

To observe the ionosphere globally and uniformly, satellite observations are ideally employed. The FORMOSAT-3/COSMIC mission (Constellation Observing System for Meteorology, Ionosphere and Climate, in short, F3/C) is initiated in 2006. The F3/C consists of 6 identical micro-satellites receiving L1-band signals from 32 GNSS satellites, probing more than $4000 \mathrm{RO}$ (radio occultation) S4-index profiles per day from $50 \mathrm{~km}$ altitude to the satellite orbit $830 \mathrm{~km}$ (Anthes et al. 2008). Using F3/C RO S4 observations for applications of the ground transportation and the aviation, Liu et al. (2016) introduce a method converting the S4max, which is the maximum value on a vertical F3/C S4-index profile, to simulate the S4-index observed on the ground. They find that the intensity pattern of the converted S4-index, S4conv, agrees well with that of the S4-index concurrently observed by co-located ground-based GNSS receivers but the S4-index has been overestimated. Based on Liu et al. (2016), Chen et al. (2017) develops an empirical model of S4conv (F3CGS4) to compute the worst-case scintillation level (or intensity) of the globe. The model predicts the S4-index when time, location, and solar activity are specified, and however, it does not provide the associated occurrence probability. In this study, we utilize the whole F3/C S4-index profile data, at least 50 times more than those of Chen et al. (2017), which shall allow us in addition to the intensity, also computing and predicting the occurrence probability of S4-index in L-band signals observed on the ground around the globe.

\section{METHODOLOGY}

The F3/C satellite sounds S4-index profile with 1 $\mathrm{Hz}$ sampling rate (i.e., $1.1-2.7 \mathrm{~km}$ altitude resolution depend on tangent heights), and denotes the maximum value, S4max, of each profile. Figure 1 depicts typical observations of S4-index profiles and their associated S4max on DOY001, 2009. The F3/C S4max frequently occurs in the Sporadic-E region at about $100 \mathrm{~km}$ altitude, and mostly scatters at $150-450 \mathrm{~km}$ altitude in the F-region. Thus, the unevenly altitudinal distribution and limited S4maxes used by Chen et al. (2017) can be significantly improved by using the whole F3/C S4-index profile that uniformly cover the global ionosphere between $80 \mathrm{~km}$ and satellite altitude at $800 \mathrm{~km}$. Hence, to uniformly sample the worst-case S4index in the vertical direction, the local maximum in each $10 \mathrm{~km}$ bin along a F3/C S4-index profile are used. The left panel of Fig. 2 depicts an arbitrary F3/C S4-index profile $\left(15.27^{\circ} \mathrm{N}, 41.42^{\circ} \mathrm{W}, 2338 \mathrm{LT}\right.$, DOY001, 2009) and the local median/maximum within a window of $\pm 5 \mathrm{~km}$ (i.e., $10 \mathrm{~km}$ ) sliding by $5 \mathrm{~km}$ altitude. Note that on the average, there are about 3 - 9 S4-indices being taken into calculation in each
$10 \mathrm{~km}$ altitudinal window (or bin).

To further examine the global three-dimensional distribution of the local S4max in various seasons and solar activities, we compute the local maximum of each profile and bind them into $3^{\circ} \times 3^{\circ} \times 10 \mathrm{~km}$ in latitude $\times$ longitude $x$ altitude during the nighttime period of $2000-0300 \mathrm{LT}$ in the solar minimum year 2009 and the solar maximum year 2013. Figure 3 illustrates maps with a $3^{\circ} \times 3^{\circ}$ in latitude $\times$ longitude resolution or $7381(=61 \times 121)$ lattices that the median of the local maximum in each bin at $100 \mathrm{~km}$ altitude (E-region) and $200-700 \mathrm{~km}$ (F-region) during nighttime (2000 - 0300LT) in the M-month (March months, \pm 45 days to spring equinox), J-month (June months, \pm 45 days to summer solstice), S-month (September months, \pm 45 days to autumn equinox), and D-month (December months, \pm 45 days to winter solstice). The pronounced pattern in the F-region generally follows the magnetic equator in the four months, taking place from $70^{\circ} \mathrm{W}$ to $0^{\circ} \mathrm{E}$ longitude (South AmericanAtlantic sector) in the D-month, from 0 to $40^{\circ} \mathrm{E}$ and 140 to $210^{\circ} \mathrm{E}$ longitude (African and Pacific sector) in the J-month, and around $0\left(60^{\circ} \mathrm{W}\right.$ to $0^{\circ} \mathrm{E}$ in the solar minimum, and $60^{\circ} \mathrm{W}$ to $60^{\circ} \mathrm{E}$ in the solar maximum) longitude (Atlantic sector) in the $\mathrm{M}$ - and S-month. In E-region (100 km altitude), the midlatitudes yield the most intense scintillation in the summer hemisphere among the four-season months. In high-latitude ionosphere, the scintillation is more intense in the northern hemisphere than in the southern hemisphere. Note that due to limited S4max being observed, the high latitude scintillation was hardly observed by Liu et al. (2016) and Chen et al. (2017). The improvement in the high latitudes suggests that the approach of this study shall have a better performance in predicting the S4-index globally.

The limb-viewing RO S4-index in the mid/low-latitude E-region is usually larger than that in the F-region because of the existence of the Sporadic-E layer, however, while the F-region is much thicker than the E-region. This results in the S4-index occurrence probability in the F-region is dominant when the S4-index (i.e., scintillation) observed from the ground. In Fig. 2a, an F3/C RO S4 profile is utilized to display the worst-case scintillation of each altitude sectors by local maxima, and the S4-index in E-region is stronger than those in the F-region. To calculate the S4conv simulating the GNSS scintillation on the ground, the calculated local maximum S4-index at every sliding $5 \mathrm{~km}$ altitudes between 80 to $800 \mathrm{~km}$ are employed,

$\mathrm{S} 4_{\text {conv }}=\sum_{\mathrm{h}=80}^{800} \mathrm{~S} 4(\mathrm{~h}) \cdot \frac{5}{\mathrm{~L}} \cdot \mathrm{dh}$

where $\mathrm{L}$ denotes the impact length in kilometer, which is the slant segment that the RO ray paths intersect in the $5 \mathrm{~km}$ tangent shell. The right bottom panel of Fig. $2 \mathrm{~b}$ displays a one-hour global distribution of S4conv during 2330 - 0030 


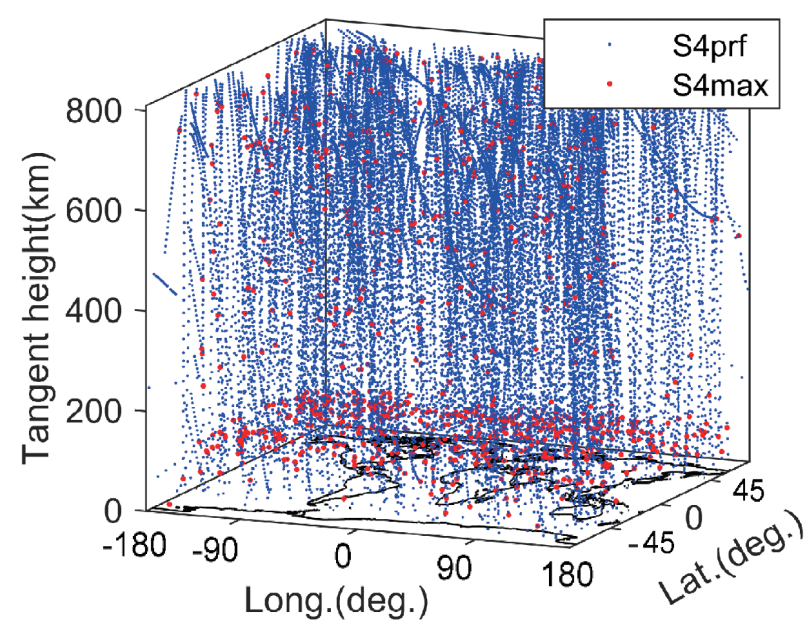

Fig. 1. F3/C S4 data distribution on DOY001, 2009. Blue dots indicate median location of S4 profile's tangent point taken every $10 \mathrm{~km}$ from 50 to $800 \mathrm{~km}$ in altitude, and red dots are S4max.
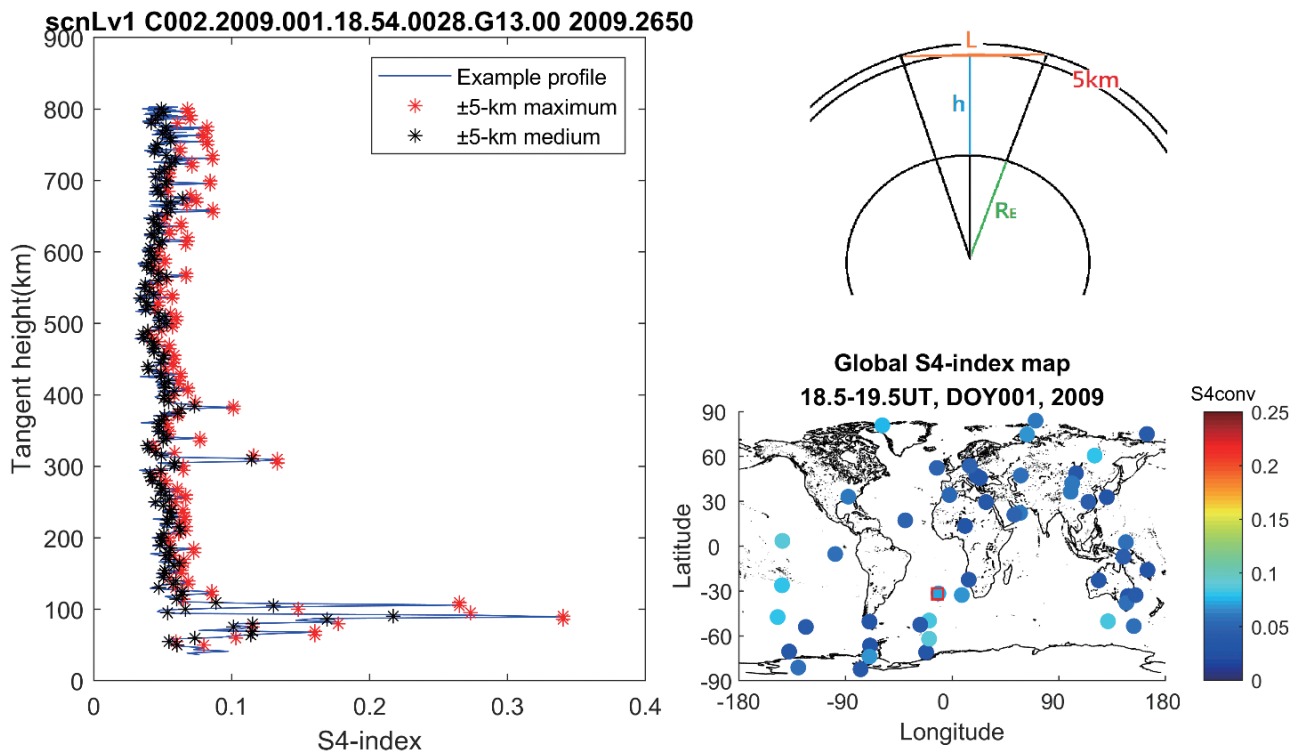

Fig. 2. An illustration deriving S4conv by F3/C S4 profile. The left panel shows the $\pm 5 \mathrm{~km}$ local altitudinal median/maximum of an arbitrary S4 profile. The top right panel is the geometry deriving S4conv, where h and $\mathrm{L}$ indicates tangent height and impact length of a 5-km shell. The bottom right panel is global distribution of the S4conv derived from the local maximums during 1830 - 1930UT on DOY001, 2009, where the red square indicates the profile in the left panel. 

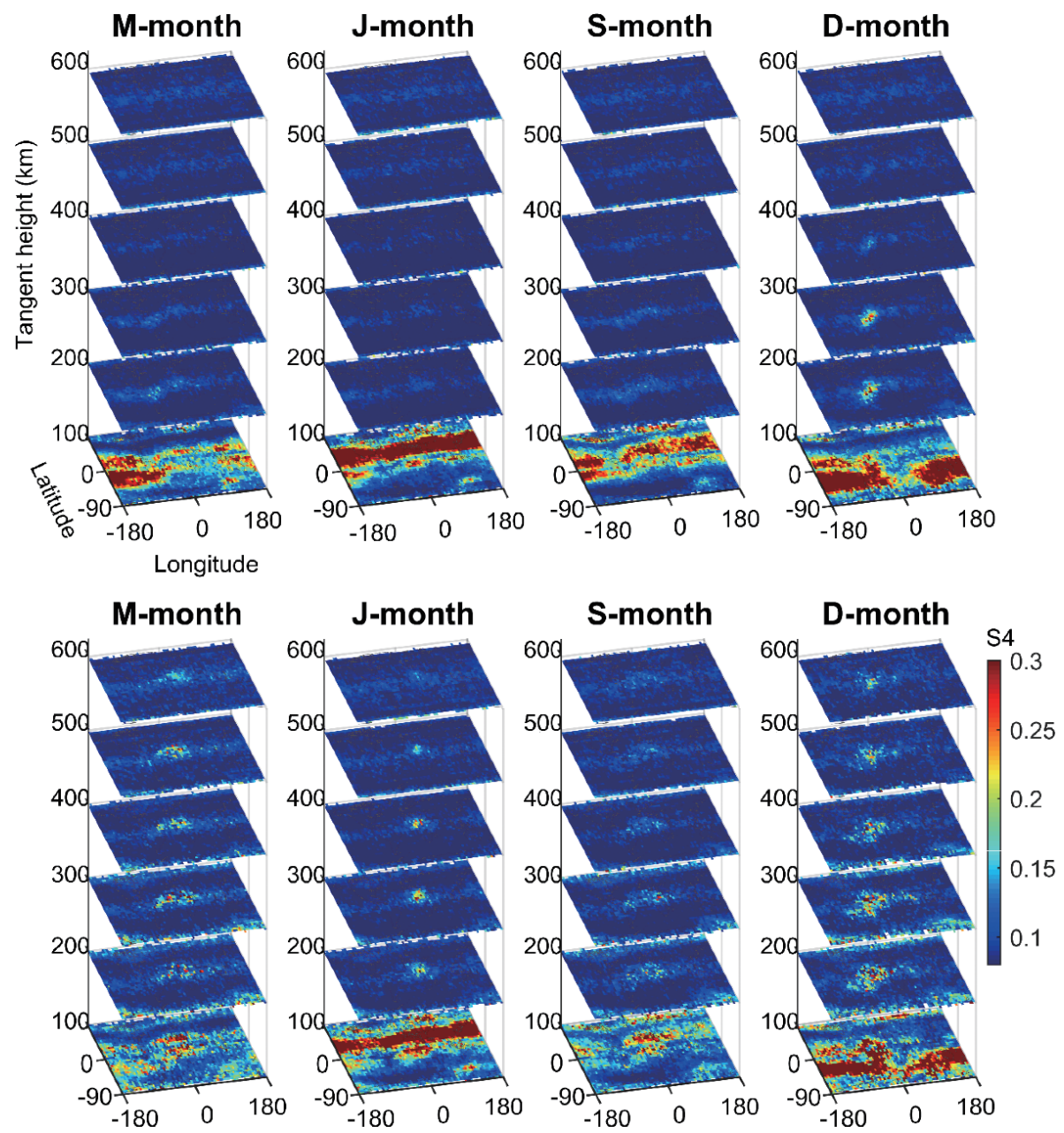

Fig. 3. Three-dimensional distributions of the $\pm 5 \mathrm{~km}$ local maximum S4 during M-month, J-month, S-month, and D-month nighttime (2000 0300LT) of year 2009 (upper panel) and 2013 (lower panel).

LT, DOY001, 2009, which displays that the S4conv in middle/high latitudes seems stronger than that in low latitudes, and the middle/high latitude S4conv yields prominent at South American sector within $180^{\circ} \mathrm{W}$ and $0^{\circ} \mathrm{E}$.

The local maximum S4-index in Fig. 3 now is further converted and integrated to obtain the S4conv by Eq. (1). The top panel of Fig. 4 depicts the median S4conv in each $3^{\circ} \times 3^{\circ}$ lattice, and the occurrence probability shown in lower panels are calculated by dividing data number above a certain S4 threshold to the overall data number in the lattice. The S4conv occurrence prominently appear mainly in low (within $\pm 20^{\circ}$ ) and high (beyond $\pm 60^{\circ}$ ) latitudes during night time (2000 - 0300 LT). This nighttime geographic scintillation distribution in high latitudes is very similar to the Rate of TEC Index (ROTI) observations reported by Cherniak et al. (2014) and Hocke et al. (2019) by using ground-based GNSS receivers. The patterns of occurrence with the given S4-index thresholds of $\mathrm{S} 4>0.1,0.15,0.2,0.25$, and 0.3 is illustrated from top to down, respectively. The pattern in occurrence probability of $\mathrm{S} 4>0.1$ and that in the $\mathrm{S} 4 \mathrm{conv}$ intensity (the top panel of Fig. 4) are similar. Note that the occurrence probability in high latitudes is greater than that in low latitudes. For $\mathrm{S} 4>0.15$, the occurrence probability in low latitudes is vice versa larger than that in high latitudes. For $\mathrm{S} 4>0.2$ to $\mathrm{S} 4>0.3$, the S4-index occurrence probabilities at high latitudes start diminishing, while those in low latitudes also get fading but the longitudinal patterns remain clear. In general, the pattern of occurrences in various thresholds (displayed in lower panels) and that of S4conv intensities (displayed in the top panel) are similar.

Since the global patterns between median S4conv and the occurrences are similar, it allows us to find the overall relationship between the intensity and assorted occurrence probabilities under various thresholds. We then draw scatter plots of the intensity and associated occurrence at each lattice for all the $7381(=61 \times 121)$ lattices under $\mathrm{S} 4>0.1$, $0.15,0.2$, and 0.25 . Figure 5 shows that the occurrence probability of each S4-index threshold is generally proportional to the scintillation intensity, while the slope of the linear fit is inversely proportion to the threshold. This agrees with 

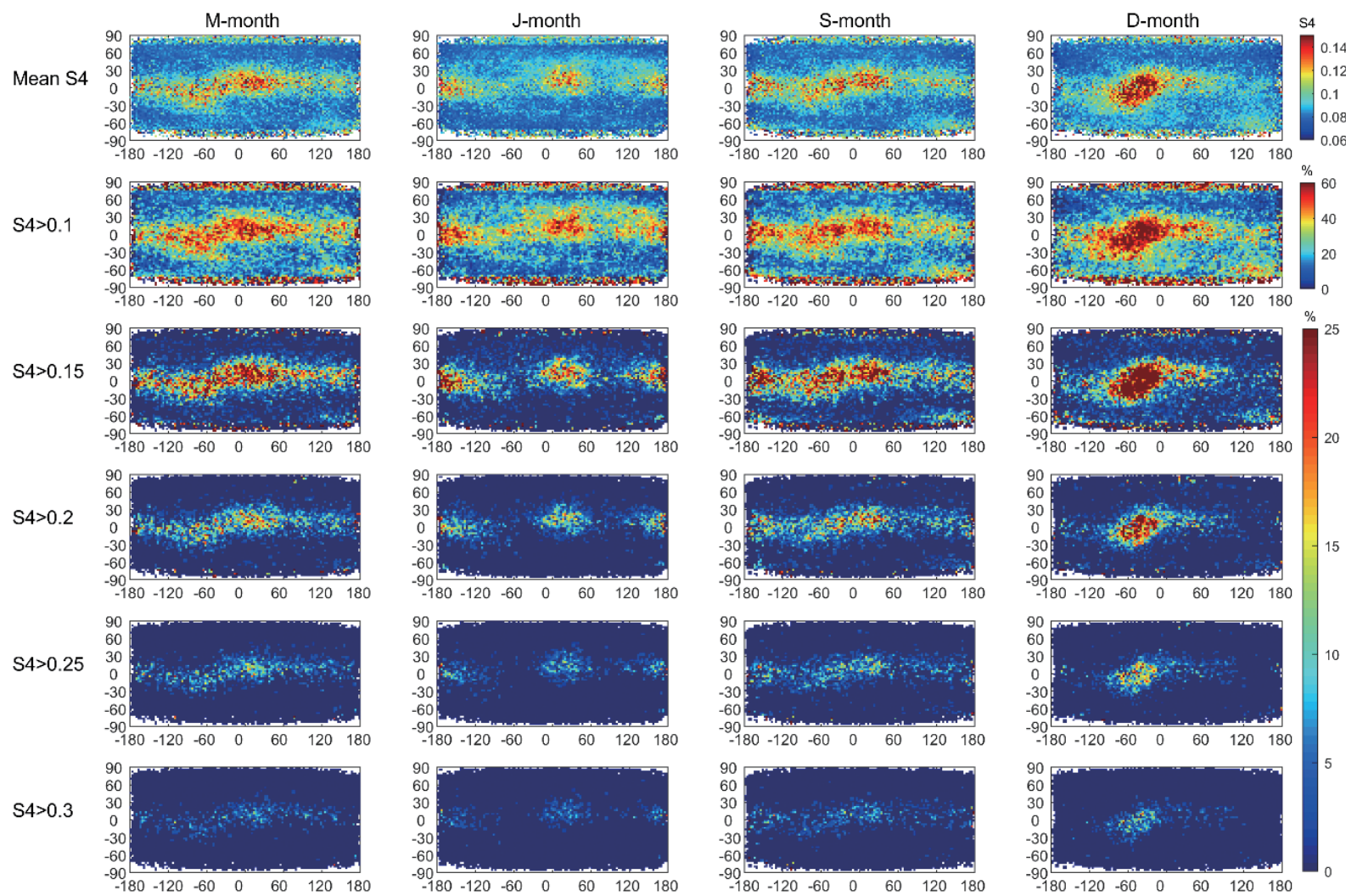

Fig. 4. A display of overall S4conv in the 4 seasons during nighttime (2000 - 0300LT) of year 2007 to 2014 (top panel). Occurrences of S4 > 0.1, > $0.15,>0.2,>0.25$, and $>0.3$ are also shown in lower panels.

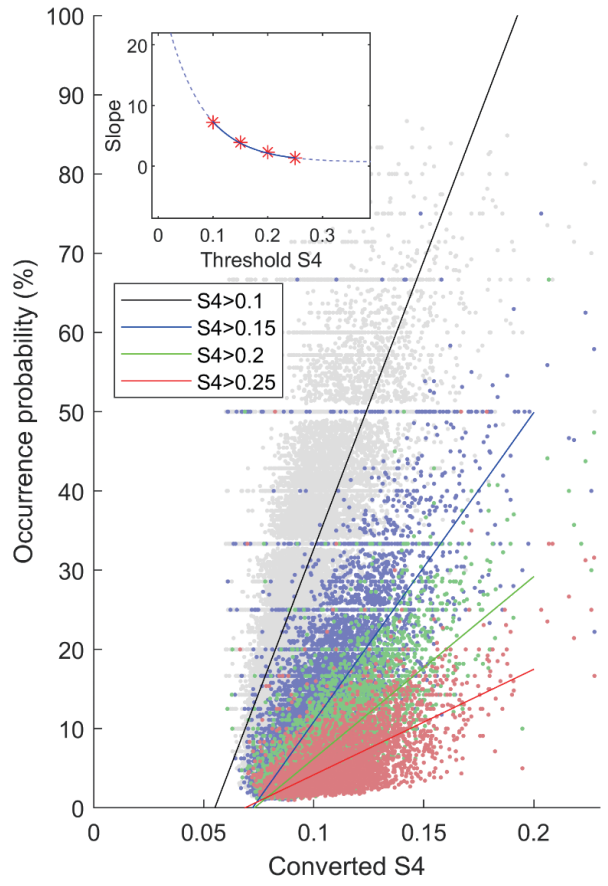

Fig. 5. S4conv intensity-occurrence distributions of S4 $>0.1$ (black dots), $>0.15$ (blue dots), $>0.2$ (green dots), and $>0.25$ (red dots). The red stars and blue lines in the upper right panel indicate the four slopes in the lower left panel and their linear fittings. 
the previous studies (cf. Basu et al. 1988) that the scintillation intensity should be proportional to its occurrence. The slopes for $\mathrm{S} 4>0.1,0.15,0.2$, and 0.25 are $7.27,3.9,2.3$, and 1.33 , respectively, which can be fitted by an exponential function expressed as (see the upright panel of Fig. 5),

Slop $=30 \cdot e^{-15 \cdot \text { Threshold S4 }}+0.65$

Note that the above slope is derived from all the 7381 (= $61 \times 121)$ lattices during the entire study period. Similar to Fig. 5, Fig. 6 displays slopes subdividing into 8 three-hour time intervals, 4-month seasons, and 8 latitude zones $(0$ $70^{\circ}$ geomagnetic dip), which are all in positive quantities, and vary with time and geomagnetic dips, as well as yield remarkable diurnal variations but hardly find seasonal variation. The slope of S4 $<0.1$ remains about large constants in all latitude zones during the daytime of $0600-1800 \mathrm{LT}$, while the slope of $\mathrm{S} 4>0.2$ is greater than 0.05 within $\pm 40^{\circ}$ geomagnetic dip during night-time of 2000 - 0300 LT.

The S4max in low latitudes are positive proportion to the solar activity (Liu et al. 2016), and the relation can be linear fitted in low solar activity years (Chen et al. 2017). Similarly, to find the relation between the S4conv occurrence probability and solar activity in low solar activity years, Fig. 7 illustrates the annual mean of nighttime (2000 - 0300 LT) S4conv occurrences within $\pm 40^{\circ}$ latitudes during 2008 to 2013. Result shows that the overall occurrence of the given S4conv thresholds are all positive linear proportion to the F10.7 index, similar to the S4max, and the slopes of the linear fit decline with the increasing S4 thresholds. Since these linear relationships have been obtained, they are used to construct and update a scintillation occurrence model based on the F3CGS4 (Chen et al. 2017), hereafter termed ROS4 model, to compute and/or predict the S4conv occurrence probability. The occurrence probability of a selected S4-index threshold is derived by multiply basis model output by the "Slope" of designated location/time. Therefore, the occurrence probability of associated scintillation intensity threshold is calculated.

\section{CURRENT MODEL RESULTS AND SCINDA OBSERVATIONS}

To validate the ROS4 model calculated occurrence probabilities, observations of 10 ground-based receivers of SCINDA in low latitudes during 2013 are employed. Figure 8 shows that for various $\mathrm{S} 4$-index thresholds the occurrence probabilities of SCINDA S4, F3/C S4conv and the ROS4 model yield good agreements. They almost simultaneously start increasing at about $1800 \mathrm{LT}$ (the probabilities of F3/C S4conv and the ROS4 model seem increasing about 1-hour faster than those of SCINDA), reach their peaks around $2100-2200 \mathrm{LT}$, begin decreasing after 2300
LT, and end by about 0100 LT (SCINDA) $0300-0600$ LT (F3/C and ROS4). In general, the three occurrence probabilities agree well with each other for $\mathrm{S} 4>0.1 \sim 0.35$, which indicates that the S4conv and the ROS4 model can be used to simulate/calculate and predict the occurrence of L-band scintillation of S4-index on the ground.

Figure 9 illustrates the model computed occurrence probability of weak scintillation $\mathrm{S} 4>0.1$. The probabilities are relatively high in the nighttime longitude sectors and move westward in low latitudes. The westward-shifted S4-index peak becomes higher in $90^{\circ} \mathrm{W}$ to $60^{\circ} \mathrm{E}$ longitude (Atlantic sector) in the M- and S-month; appears in $30^{\circ} \mathrm{W}$ to $30^{\circ} \mathrm{E}$ and 150 to $210^{\circ} \mathrm{E}$ longitude (African and Pacific sector) in the J-month, which is the lowest one among the four months; reach the annual peak in $100^{\circ} \mathrm{W}$ to $0^{\circ} \mathrm{E}$ longitude (South American-Atlantic sector) in the D-month. In total, the most prominent occurrence frequently appears over South American-Atlantic sector $\left(90^{\circ} \mathrm{W}\right.$ to $\left.60^{\circ} \mathrm{E}\right)$ during the nighttime period.

To examine the performance of the developed ROS4 model in various solar activities, the input F10.7 of the S4conv model is given 80,120 , and 160 (s.f.u.) to present the occurrence probability of scintillation response to low(F10.7 < 100), median- $(100<$ F10.7 < 140), and high-solar activity (F10.7 > 140). In Fig. 10, the S4-index occurrence probabilities of $\mathrm{S} 4>0.1$ during nighttime hour 2000 - 0300 LT are proportional to the solar activity, and the longitudinal location with high probability illustrates obvious seasonal dependence. However, there is no prominent solar activity dependence on longitudinal variation. Generally, the high S4-index occurrence probability in low latitudes is higher than $20 \%$ under low solar activity, and with higher F10.7 inputs, the occurrence probability increases to more than $40 \%$ during December months in South America longitudes. The locations of the prominent probability generally agree with those of Fig. 9 in the four months, and the occurrence patterns during high solar activity are similar to the in-situ irregularity detection of ROCSAT (Su et al. 2006) in high solar activity years. To compare the model result with latest in-situ plasma irregularity observations, the ion velocity meter (IVM) in-situ ion density measurement onboard FORMOSAT-7/COSMIC-2 (F7/C2) is utilized. Following the method of Su et al. (2006), Fig. 11 illustrates the occurrence of ion density fluctuation $(\sigma>0.3 \%)$ detected by the IVMs and the intense F7/C2 S4-index (S4 > 0.3) in F-region (150 - $550 \mathrm{~km}$ in altitude) during nighttime hours in 2020. Result shows that the latitudinal variation of the scintillation occurrence is modulate by the EIA. Chen et al. (2021) reports that stronger RO S4 (S4 > 0.5) occurs within the EPB structures intercept the EIA. Therefore, the latitudinal peak RO S4 of the model simulation in Fig. 10 and the F7/ C2 F-region RO S4-index occurrence probability in Fig. 11 both display an EIA-like double-crest structure. This distribution pattern of F7/C2 S4-index in 2020 is similar to the 

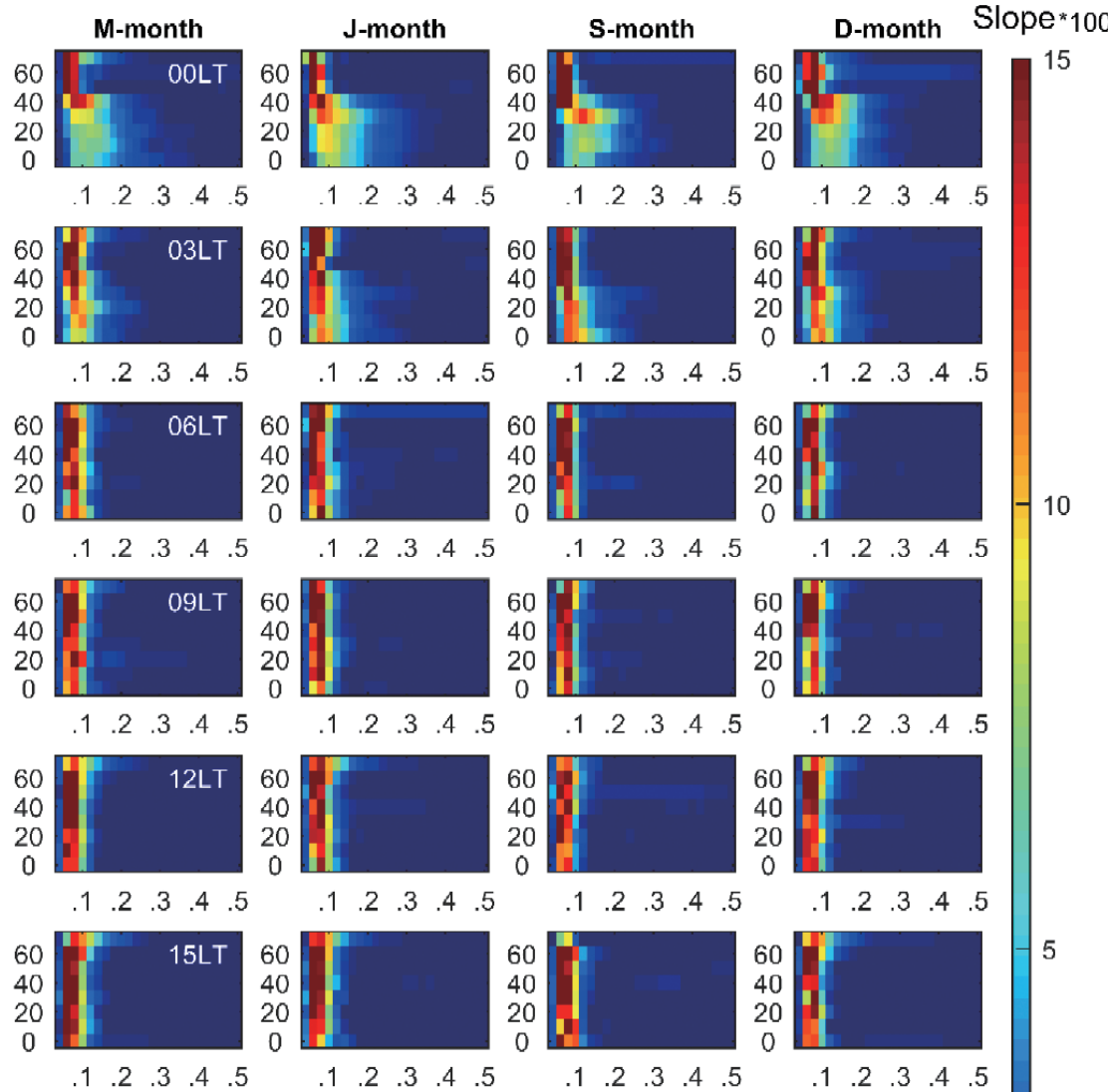

$\begin{array}{lllll}.1 & .2 & .3 & .4 & .5\end{array}$
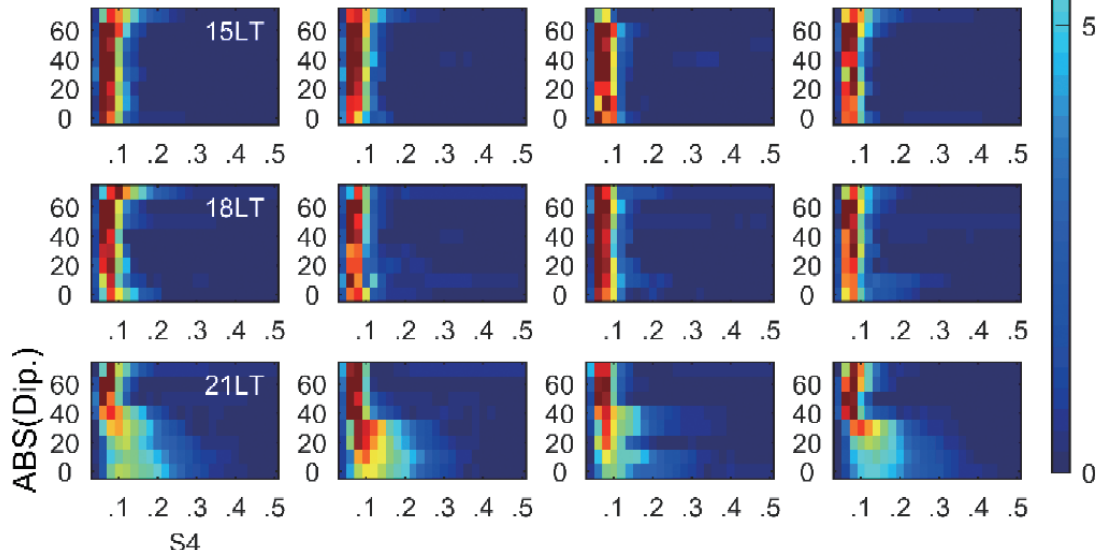

Fig. 6. The slopes of intensity-occurrence distributions in various local times, latitudes, and seasons.

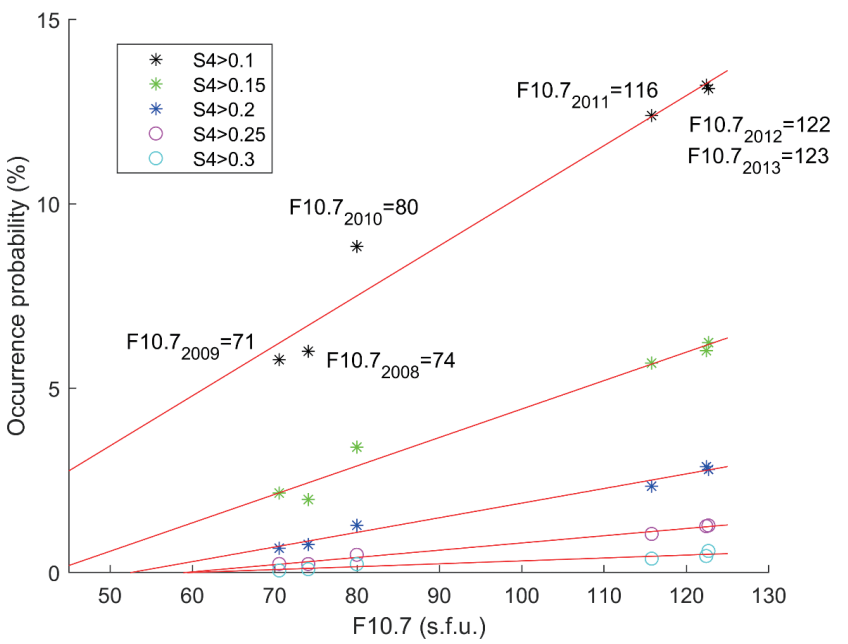

Fig. 7. The linear proportion of annual mean F10.7 and S4-index occurrence in low latitudes during 2008 to 2013. 

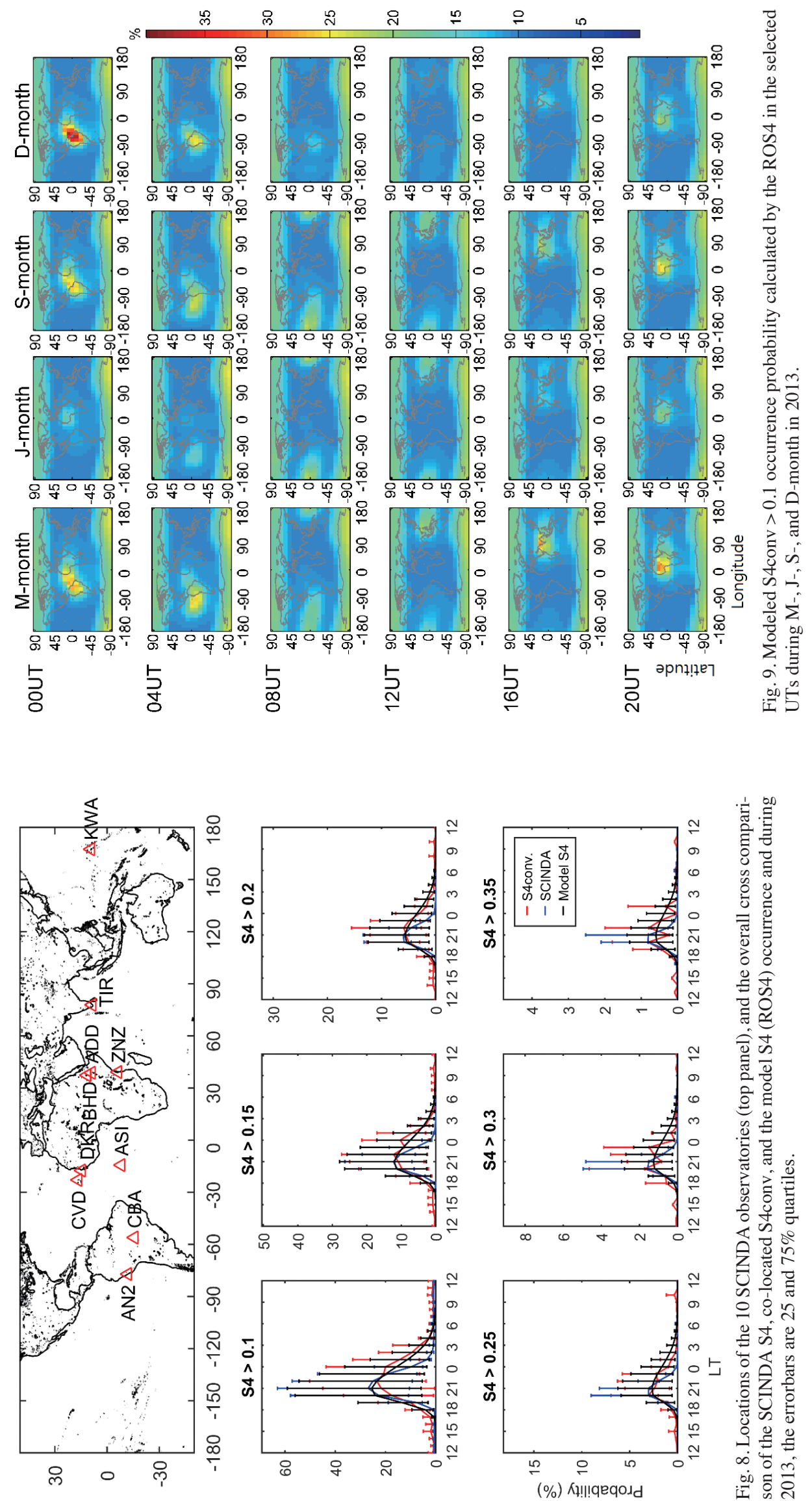

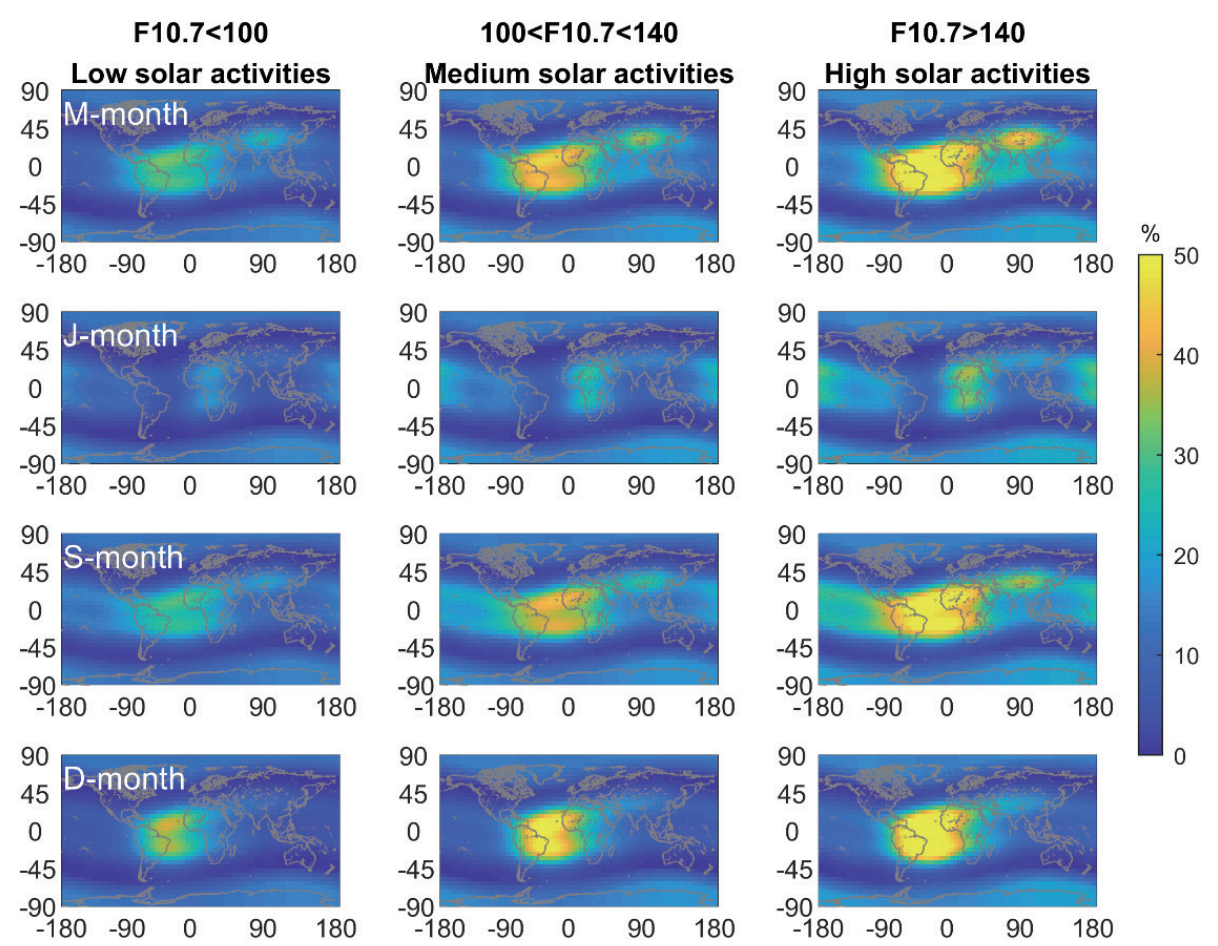

Fig. 10. Modeled S4conv $>0.1$ occurrence probability in low (the left column, F10.7 < 100), moderate (the center column, $100<$ F10.7 < 140) and high (the right column, F10.7 > 140) solar activities during nighttime hour 2000 - 0300 LT of M-, J-, S-, and D-month.

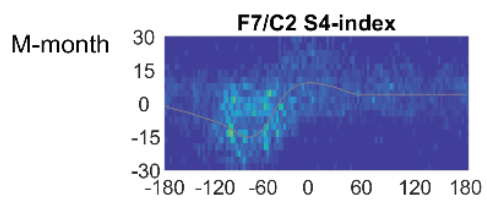

J-month
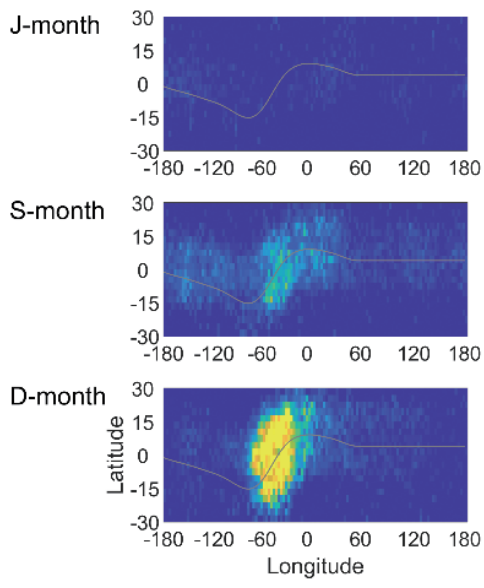
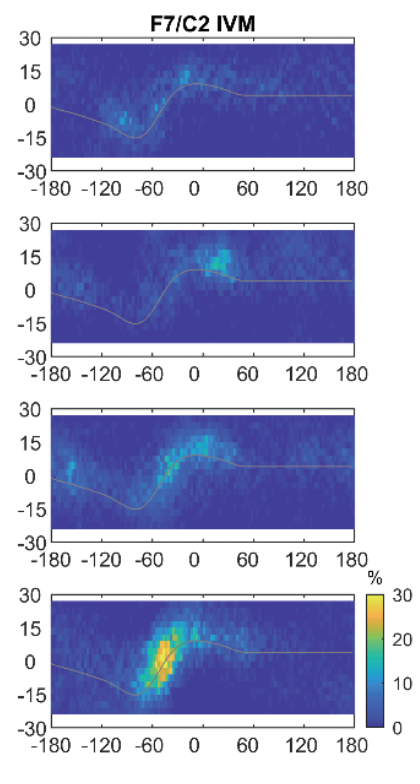
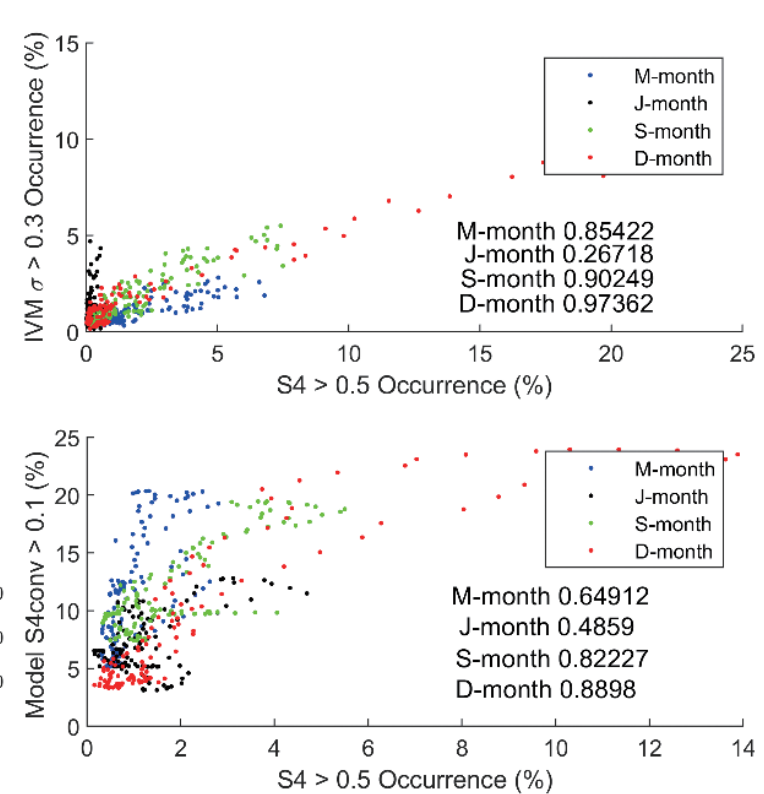

Fig. 11. F7/C2 F-region RO-S4 $>0.5$ and IVM ion density fluctuation $\sigma>0.3 \%$ (left panel) occurrence probability during nighttime hour 20000300LT in M-, J-, S-, and D-month in 2020, and the correlation analysis of the two observations (upper right panel) and the developed S4conv model (lower right panel). The blue, black, green, and red dots indicate the longitudinal distributions of the comparing patterns in M-, J-, S-, and D-month, respectively. 
model simulation during low solar activities F10.7 $<100$ in Fig. 10 (annual mean F10.7 of 2020 is 73.7). The correlation coefficients between the two occurrence probabilities above are $0.65,0.49,0.82$, and 0.89 in $\mathrm{M}-, \mathrm{J}-, \mathrm{S}-$, and D-month, respectively. On the other hand, the IVM in-situ measures ion density at $550-600 \mathrm{~km}$ altitudes in the topside ionosphere, therefore, there is no obvious double-crest structure can be found. The prominent occurrence longitudes of F7/C2 IVM and S4-index in the displayed four months are similar except for the dim F7/C2 S4-index pattern in J-month. The correlation coefficient of the longitudinal distribution in the two observation results are $0.85,0.26,0.9$, and 0.97 in $\mathrm{M}-$, $\mathrm{J}-$, S-, and D-month, respectively, indicating that the RO scintillation is majorly contributed by the developed EPBs that can be detected at the F7/C2 orbit altitude.

\section{DISCUSSION AND CONCLUSION}

This study introduces the first empirical model (ROS4) calculating ground-based scintillation occurrence of the globe by utilizing the space-based RO S4-index. The well agreements among the preliminary comparisons between ground-based S4-index of SCINDA, S4conv, and the constructed model indicate that the local maximum conversion method and the model are useful to calculate or forecast scintillation occurrence observed on the ground with given location, time, solar activity parameter, and S4-index threshold. The findings of this study are summarized as follows.

(1) Comparing to the scintillation maps constructed by the S4max (i.e., Figs. 8 and 9 in Liu et al. 2016), who's strongest scintillation tends to occur in low latitudes, the S4conv in this study show higher scintillation level and higher occurrence of weak scintillation in high latitudes. It is similar to the ground-based VHF/L-band observation of Basu et al. (1976) and Cherniak et al. (2014) that the weak scintillation occurs more often in high latitudes, indicating the S4conv derived from S4 profile performs much better than those from S4max on calculating ground-based scintillation occurrence.

(2) As the S4conv threshold increases to higher levels, the global overall ratio/slope of the relation between the scintillation intensities and associated occurrence probabilities are exponentially decreases. A more detailed investigation illustrates that the above relation is local time and latitude dependent but no obvious seasonal dependent.

(3) A preliminary comparison between the ground-based observation (SCINDA), S4conv, and the ROS4 model shows that although the hourly pattern of the three results are similar. The S4conv and the model both initiate 1 hour earlier and diminish 2 - 3 hour later than the SCINDA. The time difference may be owing to the geometry discrepancy that the integrated RO link may encounters the zonal tilted EPB bifurcations in postmidnight sector, therefore, the occurrence time of RO scintillation deviates and last longer scintillation.

(4) The pattern of scintillation S4 $>0.1$ occurrence calculated by the ROS4 is similar to the F7/C2 in-situ plasma irregularity detection at $550 \mathrm{~km}$ and F-region S4-index during nighttime hours (2000 - 0300 LT), indicating that the ground-based and RO scintillations are both contributed by F-region irregularities within developed EPBs that reaches topside ionosphere.

Acknowledgements This work was financially supported by the Center for Astronautical Physics and Engineering (CAPE) from the Featured Area Research Center program within the framework of Higher Education Sprout Project by the Ministry of Education (MOE) in Taiwan. This study is supported by the Taiwan Ministry of Science and Technology (MOST) Grant MOST 108-2119-M-008-001, 1082636-M-008-002, and 109-2636-M-008-004. The FORMOSAT-3/COSMIC S4-index scnLv1 data is available at the https://tacc.cwb.gov.tw/dataservice/fs3 cosmic2013/ level1b/scnLv1/. The authors thank Dr. Ronald Caton at Air Force Research Laboratory for providing S4-index data of SCINDA, and kindly provide suggestions of this study on the Beacon Satellite Symposium Olsztyn, Poland in 2019.

\section{REFERENCES}

Aarons, J., 1982: Global morphology of ionospheric scintillations. Proc. IEEE, 70, 360-378, doi: 10.1109/ PROC.1982.12314. [Link]

Anthes, R. A., P. A. Bernhardt, Y. Chen, L. Cucurull, K. F. Dymond, D. Ector, S. B. Healy, S.-P. Ho, D. C. Hunt, Y.-H. Kuo, H. Liu, K. Manning, C. McCormick, T. K. Meehan, W. J. Randel, C. Rocken, W. S. Schreiner, S. V. Sokolovskiy, S. Syndergaard, D. C. Thompson, K. E. Trenberth, T.-K. Wee, N. L. Yen, and Z. Zeng, 2008: The COSMIC/FORMOSAT-3 Mission: Early Results. Bull. Amer. Meteorol. Soc., 89, 313-334, doi: 10.1175/BAMS-89-3-313. [Link]

Basu, S., S. Basu, and B. K. Khan, 1976: Model of equatorial scintillations from in-situ measurements. Radio Sci., 11, 821-832, doi: 10.1029/RS011i010p00821. [Link]

Basu, S., E. MacKenzie, and S. Basu, 1988: Ionospheric constraints on VHF/UHF communications links during solar maximum and minimum periods. Radio Sci., 23, 363-378, doi: 10.1029/RS023i003p00363. [Link]

Booker, H. G., J. A. Ratcliffe, and D. H. Shinn, 1950: Diffraction from an irregular screen with applications to ionospheric problems. Philos. Trans. R. Soc.Lond.Ser. A-Math. Phys. Eng. Sci., 242, 579-607, doi: 10.1098/ rsta.1950.0011. [Link]

Carrano, C. S. and C. L. Rino, 2016: A theory of scintillation for two-component power law irregularity spectra: Overview and numerical results. Radio Sci., 51, 
789-813, doi: 10.1002/2015RS005903. [Link]

Chen, S.-P., D. Bilitza, J.-Y. Liu, R. Caton, L. C. Chang, and W.-H. Yeh, 2017: An empirical model of L-band scintillation S4 index constructed by using FORMOSAT-3/COSMIC data. Adv. Space Res., 60, 10151028, doi: 10.1016/j.asr.2017.05.031. [Link]

Chen, S.-P., C. C. H. Lin, P. K. Rajesh, J.-Y. Liu, R. Eastes, M.-Y.Chou, and J.-M. Choi, 2021: Near real-time global plasma irregularity monitoring by FORMOSAT-7/ COSMIC-2. J. Geophys. Res., 126, e2020JA028339, doi: 10.1029/2020JA028339. [Link]

Cherniak, I., A. Krankowski, and I. Zakharenkova, 2014: Observation of the ionospheric irregularities over the Northern Hemisphere: Methodology and Service. Radio Sci., 49, 653-662, doi: 10.1002/2014RS005433. [Link]

Hewish, A., 1951: The diffraction of radio waves in passing through a phase-changing ionosphere. Proc. R. Soc. Lond. A, 209, 81-96, doi: 10.1098/rspa.1951.0189. [Link]

Hocke, K., H. Liu, N. Pedatella, and G. Ma, 2019: Global sounding of $\mathrm{F}$ region irregularities by COSMIC during a geomagnetic storm. Ann. Geophys., 37, 235-242, doi: 10.5194/angeo-37-235-2019. [Link]

Liu, J. Y., S. P. Chen, W. H. Yeh, H. F. Tsai, and P. K. Rajesh, 2016: Worst-case GPS scintillations on the ground estimated from radio occultation observations of FORMOSAT-3/COSMIC during 2007-2014. Surv. Geophys., 37, 791-809, doi: 10.1007/s10712-0159355-X. [Link]

Secan, J. A., R. M. Bussey, E. J. Fremouw, and S. Basu, 1995: An improved model of equatorial scintillation. Radio Sci., 30, 607-617, doi: 10.1029/94RS03172. [Link]

Sreeja, V. and M. Aquino, 2014: Statistics of ionospheric scintillation occurrence over European high latitudes. J. Atmos. Sol.-Terr. Phys., 120, 96-101, doi: 10.1016/j. jastp.2014.09.003. [Link]

Su, S. -Y., C. H. Liu, H. H. Ho, and C. K. Chao, 2006: Distribution characteristics of topside ionospheric density irregularities: Equatorial versus midlatitude regions. J. Geophys. Res., 111, A06305, doi: 10.1029/2005JA011330. [Link]

Yeh, K. C. and C.-H. Liu, 1982: Radio wave scintillations in the ionosphere. Proc. IEEE, 70, 324-360, doi: 10.1109/ PROC.1982.12313. [Link]

Zhang, H., Y. Liu, J. Wu, T. Xu, and D. Sheng, 2015: Observations and modeling of UHF-band scintillation occurrence probability over the low-latitude region of China during the maximum activity of solar cycle 24. Ann. Geophys., 33, 93-100, doi: 10.5194/angeo-33-93-2015. [Link] 